\title{
Maximum-Revenue Multicast Routing and Partial Admission Control for Multirate Multimedia Distribution
}

\author{
Hsu-Chen Cheng and Frank Yeong-Sung Lin \\ Department of Information Management, National Taiwan University \\ \{d7725002,yslin\}@im.ntu.edu.tw
}

\begin{abstract}
In this paper, we intend to solve the problem of maximum-revenue multicast routing with a partial admission control mechanism for multirate multimedia distribution. Specifically, for a given network topology, a given link capacity, the destinations of a multicast group, and the bandwidth requirement of each destination, we attempt to find a feasible routing solution to maximize the revenue of the multicast trees. The partial admission control mechanism means that the admission policy of the multicast group will not be based on a traditional "all or none" strategy. Instead it considers accepting of partial portions of destinations for the requested multicast group. Firstly, we model this problem as an optimization problem. Then, we propose a simple heuristic algorithm and an optimization based heuristic to solve this problem. The methodology taken for solving the problem is Lagrangean relaxation. Computational experiments have been performed on regular networks, random networks, and scale-free networks.
\end{abstract}

\section{Introduction}

In order to support the advanced applications such as e-learning and video conference, it will be necessary for the service delivery infrastructure to provide multimedia services and multicast data delivery within guaranteed bounds of Quality-of-Service (QoS). Multimedia application environments are characterized by large bandwidth variations due to the heterogeneous access technologies of networks and different receivers' quality requirements, which make it difficult to achieve bandwidth efficiency and service flexibility. There are many challenging issues that need to be addressed in designing architectures and mechanisms for multicast data transmission [1].

Taking advantage of recent advances in video encoding and transmission technologies, either by a progress coder [2] or video gateway [3] [4], different destinations can request a different bandwidth requirement from the source, after which the source only needs to transmit signals that are sufficient for the highest bandwidth destination into a single multicast tree. A multi-layered or multirate encoder encodes video data into more than one video stream, including one base layer stream and several enhancement layer streams. The base layer contains the most important portions of the video stream for achieving the minimum quality level. The enhancement layers contain the other portions of the video stream for refining the quality of the base layer stream. Reference [5] discusses the min-cost routing problem of multirate multicasting.

In order to meet the requirements for multimedia distribution, network operators invest more and more capital to enlarge their network capacity. In addition to enlarging the network capacity, there is still one way to achieve the goal of revenue maximization, namely: network planning or traffic engineering. Traffic engineering is the process of controlling how traffic flows through a network in order to optimize resource utilization and network performance. At the same time, it can provide QoS. Admission control is often considered a by-product of QoS routing and resource reservation. If the latter is successfully performed along the route(s) selected by the routing algorithm, the connection request is accepted; otherwise, it is rejected. It is clear from the above introduction to know that in order to consider the QoS assurance issue, the three closely-related mechanisms of admission control, routing and resource reservation should be treated jointly.

In this paper, we jointly considering the above three mechanisms and intend to solve the problem of maximum-revenue multicast routing with a partial admission control mechanism for multirate multimedia distribution. For multirate video distribution, which is different from the conventional Steiner tree problem, each receiver can request a different quality of video. This means that each link's flow of the multicast tree is different and is dependent on the maximum rate of the receiver sharing the link. The partial admission control mechanism means that the admission policy of the 
multicast group will not be based on a traditional "all or none" strategy. Instead it considers accepting of partial portions of destinations for the requested multicast group. More specifically, for a given network topology, a given link capacity, the destinations of a multicast group, and the bandwidth requirement of each multicast node, we attempt to find a feasible admission decision and routing solution to maximize the revenue of the multicast trees.

The rest of this paper is organized as follows. In Section 2, we formally define the problem being studied, as well as a mathematical formulation of maxrevenue optimization is proposed. Section 3 applies Lagrangean relaxation as a solution approach to the problem. Section 4, illustrates the computational experiments. Finally, in Section 5 we present our conclusions and the direction of future research.

\section{Problem formulation}

The network is modeled as a graph where the switches are depicted as nodes and the links are depicted as arcs. A user group, which has one source and one or more destinations, is an application requesting transmission on this network. Given the network topology, the capacity of links and the bandwidth requirement of every destination of a user group, we want to jointly determine the following decision variables: (1) the routing assignment (a tree for multicasting, or path for unicasting) of each admitted destination; and (2) the admitted number of destinations of each partially admitted multicast group. We assume that the multicasting is multirate.

Table 1. Description of notations

\begin{tabular}{|c|l|}
\hline \multicolumn{2}{|c|}{ Given Parameters } \\
\hline Notation & Descriptions \\
\hline$F_{g q}$ & $\begin{array}{l}\text { Revenue generated from admitting partial users } \\
\text { of multicast group } g \text { with propriety } q \text {, which is } \\
\text { a function of } f_{g q} \text { and } a_{g q}\end{array}$ \\
\hline$a_{g q}$ & $\begin{array}{l}\text { Revenue generated from admitting multicast } \\
\text { group } g \text { with propriety } q\end{array}$ \\
\hline$\alpha_{g d}$ & $\begin{array}{l}\text { Traffic requirement of destination } d \text { multicast } \\
\text { group } g\end{array}$ \\
\hline$G$ & The set of all multicast groups \\
\hline$V$ & The set of nodes in the network \\
\hline$L$ & The set of links in the network \\
\hline$Q$ & The set of priorities in the network \\
\hline$D_{g}$ & The set of destinations of multicast group $g$ \\
\hline$T_{g q}$ & $\begin{array}{l}\text { The set of destinations of priority } q \text { in multicast } \\
\text { group } g\end{array}$ \\
\hline$C_{l}$ & Capacity of link $l$ \\
\hline$I_{v}$ & The incoming links to node $v$ \\
\hline$r_{g}$ & The multicast root of multicast group $g$ \\
\hline
\end{tabular}

\begin{tabular}{|c|l|}
\hline$I_{r_{g}}$ & The incoming links to node $r_{g}$ \\
\hline$P_{g d}$ & $\begin{array}{l}\text { The set of paths user } d \text { of multicast group } g \\
\text { may use }\end{array}$ \\
\hline$\delta_{p l}$ & $\begin{array}{l}\text { The indicator function which is } 1 \text { if link } l \text { is on } \\
\text { path } p \text { and } 0 \text { otherwise }\end{array}$ \\
\hline$\sigma_{q g}$ & $\begin{array}{l}\text { The indicator function which is } 1 \text { if priority } q \text { is } \\
\text { selected for destination } d \text { and } 0 \text { otherwise }\end{array}$ \\
\hline & \multicolumn{1}{c|}{ Decision Variables } \\
\hline Notation & Descriptions \\
\hline$x_{g p d}$ & $\begin{array}{l}1 \text { if path } p \text { is selected for group } g \text { destined for } \\
\text { destination } d \text { and } 0 \text { otherwise. }\end{array}$ \\
\hline$y_{g l}$ & $\begin{array}{l}1 \text { if link } l \text { is on the subtree adopted by multicast } \\
\text { group } g \text { and } 0 \text { otherwise. }\end{array}$ \\
\hline$m_{g l}$ & $\begin{array}{l}\text { The maximum traffic requirement of the } \\
\text { destination in multicast group } g \text { that are } \\
\text { connected to the source through link } l .\end{array}$ \\
\hline$f_{g q}$ & $\begin{array}{l}\text { The number of admitted destinations of priority } \\
q \text { in multicast group } g .\end{array}$ \\
\hline
\end{tabular}

By formulating the problem as a mathematical programming problem, we intend to solve it optimally to obtain a network that fits into our goal, i.e., ensures the network operator can earn maximum revenue from servicing the partially admitted destinations.

This model is based on the following viable assumptions.

- The revenue from each partially admitted group can be fully characterized by two parameters: the entire admitted revenue of the group associated with specific priority and the number of admitted destinations of specific priority.

- The revenue from each partially admitted group associated with specific priority is a monotonically increasing function with respect to the number of admitted destinations of specific priority.

- The revenue function from each partially admitted group associated with specific priority is a concave function with respect to the entire admitted revenue of the group associated with specific priority and the number of admitted destinations of specific priority. However, the entire admitted revenue and the number of admitted destinations jointly may not be a concave function.

- The revenue from each partially admitted group associated with specific priority is independent.

The notations used to model the problem are listed in Table 1.

\section{Optimization Problem:}

\section{Objective function:}

$$
\min -\sum_{g \in G} \sum_{q \in Q} F_{g q}\left(a_{g q}, f_{g q}\right)
$$

subject to:

$$
\sum_{p \in P_{g d}} \alpha_{g d} x_{g p d} \delta_{p l} \leq m_{g l} \quad \forall g \in G, d \in D_{g}, l \in L
$$




$$
\begin{array}{ll}
\sum_{g \in G} m_{g l} \leq C_{l} & \forall l \in L \\
m_{g l} \in\left[0, \max _{d \in D} \alpha_{g d}\right] & \forall g \in G, l \in L \\
\sum_{l \in L} y_{g l} \geq \sum_{d \in D_{g}} \sum_{p \in P_{g d}} x_{g p d} & \forall g \in G \\
\sum_{d \in D_{g}} \sum_{p \in P_{g d}} x_{g p d} \delta_{p l} \leq\left|D_{g}\right| y_{g l} & \forall g \in G, l \in L \\
\sum_{l \in I_{v}} y_{g l} \leq 1 & \forall g \in G, v \in V-\left\{r_{g}\right\} \\
\sum_{l \in I_{r_{g}}} y_{g l}=0 & \forall g \in G \\
x_{g p d}=0 \text { or } 1 & \forall g \in G, p \in P_{g d}, d \in D_{g} \\
\sum_{d \in D_{g}} \sum_{p \in p_{g d}} x_{g p d} \sigma_{q d}=f_{g q} & \forall g \in G, q \in Q \\
f_{g q} \in\left\{0,1,2, \ldots \ldots,\left|T_{g q}\right|\right\} & \forall g \in G, q \in Q \\
y_{g l}=0 \text { or } 1 & \forall l \in L, g \in G \\
\sum_{p \in P_{g d}} x_{g p d} \leq 1 & \forall g \in G, d \in D_{g}
\end{array}
$$

The objective function of (IP) is to maximize the total revenue $F_{q g}$ of servicing the partially admitted destinations in multicast group $g$ associated with specific priority, where $g \in G, q \in Q$ and $G$ is the set of user groups requesting transmission. $F_{g q}$ reflects the priority of partial users belonging to group $g$, while different choices of $F_{g q}$ may provide different physical meanings of the objective function. For example, if $F_{\mathrm{g} q}$ is chosen to be the mean traffic requirement of partial users belonging to group $g$ associated with priority $q$, then the objective function is to maximize the total system throughput. In general, if user group $g$ with priority $q$ is to be given a higher importance, then the corresponding $F_{g q}$ may be assigned a larger value.

Constraint (1) and (2) are the capacity constraint. In this model, the variable $m_{g l}$ can be viewed as the estimate of the aggregate flows. Since the object function is strictly decreasing with $m_{g l}$ and (IP) is a maximization problem, each $m_{g l}$ will exactly equal to the aggregate flow in an optimal solution. Constraint (3) is a redundant constraint which provides upper and lower bounds on the maximum traffic requirement for multicast group $g$ on link $l$. Constraint (4) requires that if one path is selected for group $g$ destined for destination $d$, it must also be on the subtree adopted by multicast group $g$. Constraint (5) is the tree constraint, which requires that the union of the selected paths for the destinations of user group $g$ forms a tree. Constraints (4) and (6) require that the number of selected incoming links $y_{g l}$ is 1 or 0 and each node, excepting the root, has only one incoming link. Constraint (6) requires that the number of selected incoming link $y_{g l}$ to node is 1 or 0 . Constraint (7) requires that there is no selected incoming link $y_{g l}$ that is the root of multicast group $g$. As a result, the links we select can form a tree. Constraint (8) and (12) require that at most one path is selected for each admitted multicast source-destination pair, while Constraint (9) relates the routing decision variables $x_{g p d}$ to the auxiliary variables $f_{g q}$. Constraint (10) requires that the number of admitted destinations in multicast group $g$ with priority $q$ is the set of integers.

\section{Solution procedure}

\subsection{Lagrangean relaxation}

Lagrangean method has become one of the best tools for optimization problems such as integer programming, linear programming combinatorial optimization, and non-linear programming [6]. By adjusting the multiplier of Lagrangean relaxation, we can obtain the upper and lower bounds of this problem. The Lagrangean multiplier problem can be solved in a variety of ways. The subgradient optimization technique is possibly the most popular technique [6] [7]. By using the Lagrangean Relaxation method, we can transform the primal problem (IP) into the following Lagrangean Relaxation problem (LR) where Constraints (1) (4) (5) and (9) are relaxed. For a vector of Lagrangean multipliers, a Lagrangean Relaxation problem of (IP) is given by

\section{Optimization problem (LR):}

$$
\begin{aligned}
& Z_{D}(\beta, \lambda, \theta, \varepsilon)= \\
& \min -\sum_{g \in G} \sum_{q \in Q} F_{g q}\left(a_{g q}, f_{g q}\right)+\sum_{g \in G} \sum_{l \in L} \sum_{d \in D_{g}} \sum_{p \in P_{g d}} \beta_{g d l} \alpha_{g d} x_{g p d} \delta_{p l} \\
&-\sum_{g \in G} \sum_{d \in D_{g}} \sum_{l \in L} \beta_{g d l} m_{g l}+\sum_{g \in G} \sum_{l \in L} \lambda_{g} y_{g l}-\sum_{g \in G} \sum_{d \in D_{g}} \sum_{p \in P_{g d}} \lambda_{g} x_{g p d} \\
&+\sum_{g \in G} \sum_{l \in L} \sum_{d \in D_{g}} \sum_{p \in P_{g d}} \theta_{g l} x_{g p d} \delta_{p l}-\sum_{g \in G} \sum_{l \in L} \theta_{g l}\left|D_{g}\right| y_{g l} \\
&+\sum_{g \in G} \sum_{q \in Q} \sum_{d \in D_{g}} \sum_{p \in P_{g d}} \varepsilon_{g p} x_{g p d} \sigma_{q d}-\sum_{g \in G} \sum_{q \in Q} \varepsilon_{g q} f_{g q}
\end{aligned}
$$

subject to: $(2)(3)(6)(7)(8)(10)(11)(12)$

where $\beta_{g d l}, \lambda_{g}, \theta_{g l}$ and $\varepsilon_{g q}$ are Lagrangean multipliers and $\beta_{g d}, \theta_{g l} \geq 0$. To solve (LR), we can decompose (LR) into the following four independent and easily solvable optimization subproblems.

Subproblem 1: (related to decision variable $x_{g p d}$ ) $Z_{\text {Sub1 }}(\beta, \lambda, \theta, \varepsilon)=$

$\min \sum_{g \in G} \sum_{d \in D_{g}} \sum_{p \in P_{g d}}\left(\sum_{l \in L} \delta_{p l}\left(\beta_{g d l} \alpha_{g d}+\theta_{g l}\right)+\sum_{q \in Q} \varepsilon_{g} \sigma_{g d}-\lambda_{g}\right) x_{g p d}$ subject to: $(8)(12)$.

The Subproblem 1 is to determine $x_{g p d}$ and it can be further decomposed into $|G|\left|D_{g}\right|$ independent shortest path problems with nonnegative arc weights $\beta_{g d l} \alpha_{g d}+\theta_{g l}$. If the shortest cost plus coefficient $\sum_{q \in Q} \varepsilon_{g} \sigma_{g d}-\lambda_{g}$ is no 
more than 0 , than assign the corresponding $x_{g p d}$ to 1 , and 0 otherwise.

Subproblem 2: (related to decision variable $y_{g l}$ )

$$
Z_{S u b 2}(\lambda, \theta)=\min \sum_{g \in G} \sum_{l \in L}\left(\lambda_{g}-\theta_{g l}\left|D_{g}\right|\right) y_{g l}
$$

subject to: $(6)(7)(11)$.

The Subproblem 2 can be decomposed into $|\mathrm{G}|$ independent problems. The algorithm to solve to Subproblem 2 is stated as follows:

1. Compute the coefficient $\lambda_{g}-\theta_{g l}\left|D_{g}\right|$ for all links in the multicast group $g$.

2. Sort the links in ascending order according to the coefficient.

3. According to the order and complying with constraints (6) and (7), if the coefficient is less than zero, assigns the corresponding negative coefficient of $y_{g l}$ to 1 ; otherwise 0 .

Subproblem 3: (related to decision variable $f_{g q}$ )

$$
Z_{\text {Sub3 }}(\varepsilon)=\min -\sum_{g \in G} \sum_{q \in Q}\left(F_{g q}\left(a_{g q}, f_{g q}\right)+\varepsilon_{g q} f_{g q}\right)
$$

subject to: (10).

We can easily solve Subproblem 3 optimally by exhaustively searching from the known set of $f_{g q}$.

Subproblem 4: (related to decision variable $m_{g l}$ )

$$
Z_{\text {Sub4 }}(\beta)=\min \sum_{l \in L}\left(\sum_{g \in G}\left(-\sum_{d \in D_{g}} \beta_{g d l}\right) m_{g l}\right)
$$

subject to: (2)(3).

We can decompose Subproblem 4 into $|\mathrm{L}|$ independent problems. The algorithm to solve Subproblem 4 is:

Step 1 Compute $-\sum_{d \in D_{g}} \beta_{g d l}$ for link $l$ of multicast group $g$.

Step 2 Sort the negative coefficient $-\sum_{d \in D_{g}} \beta_{g d l}$ from the smallest value to the largest value

Step 3 According the sorted sequence. $<\mathrm{i}>$ assigns the corresponding $m_{g l}$ to the maximum traffic requirement in the multicast group and adds to the sum value until the total amount of maximum traffic requirements on link $l$ is less than the capacity of link $l$. <ii $>$ assign the boundary negative coefficient of $m_{g l}$ to the difference between the capacity on link $l$ and the sum value of $m_{g l},<$ iii $>$ assign the other coefficients of $m_{g l}$ to 0 .

According to the weak Lagrangean duality theorem [6], for any $\beta_{g d l}, \theta_{g l} \geq 0, Z_{D}\left(\beta_{g d l}, \lambda_{g}, \theta_{g l}, \varepsilon_{g q}\right)$ is a lower bound on $Z_{I P}$. The following dual problem (D) is then constructed to calculate the tightest lower bound.

\section{Dual Problem (D):}

$$
Z_{D}=\max Z_{\mathrm{D}}\left(\beta_{g d l}, \lambda_{g}, \theta_{g l}, \varepsilon_{g q}\right)
$$

subject to: $\beta_{\text {gdl }}, \theta_{g l} \geq 0$.

There are several methods for solving the dual problem (D). The most popular is the subgradient method [8], which is employed here.

\subsection{Getting primal feasible solutions}

After optimally solving the Lagrangean relaxation problem, we get a set of decision variables. However, this solution would not be a feasible one for the primal problem since some of constraints are not satisfied. Thus, minor modification of decision variables, or the hints of multipliers must be taken, to obtain the primal feasible solution of problem (IP). Generally speaking, the best primal feasible solution is an upper bound (UB) of the problem (IP), while the Lagrangean dual problem solution guarantees the lower bound (LB) of problem (IP). Iteratively, by solving the Lagrangean dual problem and getting the primal feasible solution, we get the LB and UB, respectively. So, the gap between UB and LB, computed by (UBLB) $/ \mathrm{LB}^{*} 100 \%$, illustrates the optimality of problem solution. The smaller gap computed, the better the optimality.

Here we propose a comprehensive, two-part method to obtain a primal feasible solution. It utilized a Lagrangean multipliers based heuristic, followed by adjustment procedures. While solving the Lagrangean relaxation dual problem, we may get some multipliers related to each OD pair and links. According to the information, we can make our routing more efficient. We describe the Lagrangean based heuristic below.

\section{[Lagrangean Multipliers based heuristic]}

Step $1 U$ se $\lambda_{g}-\theta_{g l}\left|D_{g}\right|$ as link l's arc weight and run the M-T-M heuristic [10] to get a spanning tree for each multicast group.

Step 2 Drop procedures:

2.1 Check the capacity constraint of each link. If there is a link violate the capacity constraint, go to Step 2.2, otherwise Step 3.

2.2 Sort the links in descending order according to $\left\{C_{l}\right.$ - the aggregate flow on the link $\}$. Choose the maximal overflow link and drop the group with the maximal subgradient $\left(-F_{g q}\left(a_{g q}, f_{g q}\right)-\varepsilon_{g q} f_{g q}\right)$. Go to Step 2.1.

Step 3 Add procedures:

3.1 Sort the dropped group in ascending order according to the subgradient $\left(-F_{g q}\left(a_{g q}, f_{g q}\right)\right.$ $\varepsilon_{g q}\left(f_{g q}\right)$.

3.2 In accordance with the order, re-add the groups to the network. Use $\lambda_{g}-\theta_{g l}\left|D_{g}\right|$ as 
link l's arc weight, removes the overflow links from the graph and run the M-T-M heuristic. If it can not find a route for the destinations, drop the destinations.

\section{Computational experiments}

In this section, computational experiments on the Lagrangean relaxation based heuristic and other primal heuristics are reported. The heuristics are tested on three kinds of networks - regular networks, random networks, and scale-free networks.

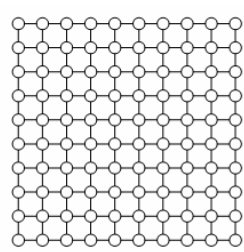

(a) grid network

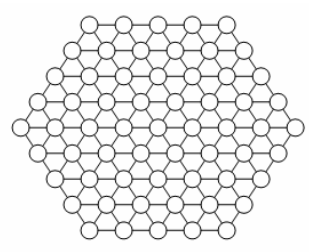

(b) cellular network

\section{Figure 1. Regular networks}

Two regular networks shown in Figure 1 are tested in our experiment. The first one is a grid network that contains 100 nodes and 180 links, and the second is a cellular network containing 61 nodes and 156 links. Random networks tested in this paper are generated randomly, each having 100 nodes. The candidate links between all node pairs are given a probability following the uniform distribution. In the experiments, we link the node pair with a probability smaller than $2 \%$. Reference [9] shows that the scale-free networks can arise from a simple dynamic model that combines incremental growth with a preference for new nodes to connect to existing ones that are already well connected. In our experiments, we applied this preferential attachment method to generate the scalefree networks. The number of nodes in the testing networks is 100 .

In order to prove that our heuristics are good enough, we also implement a simple algorithm to compare with our heuristic.

\section{[Simple Algorithm]}

Step 1 Set link $l$ 's arc weight to 1 and run the M-T-M heuristic to get a spanning tree for each multicast group.

\section{Step 2 Drop procedures:}

2.1 Check the capacity constraint of each link. If there is a link violate the capacity constraint, go to Step 2.2, otherwise Step 3.

2.2 Sort the links in descending order according to $\left\{C_{l}\right.$ - the aggregate flow on the link\}. Choose the maximal overflow link and drop the nodes of group which have maximum flow on that link. Go to Step 2.1.

Step 3 Add procedures:

3.1 Sort the dropped group in ascending order according to the group ID and node ID.

3.2 In accordance with the order, re-add the groups to the network. Remove the overflow links from the graph, set each link's arc weight to the aggregate flow of the link and run the M-T-M heuristic. If it can not find a feasible route for the destinations, drop the destinations.

For each testing network, several distinct cases, which have different pre-determined parameters such as the link capacity, the number of multicast group and the number of nodes in a group, are considered. The traffic demands for each multicast group are drawn from a random variable uniformly distributed in prespecified categories $\{1,2,5,10,15,20\}$. We conducted 120 experiments for each kind of network. For each experiment, the result was determined by the group source and destinations generated randomly. Table 2 summaries the selected results of the computational experiments. For each testing network, the maximum improvement ratio between the simple heuristic and the Lagrangean based heuristic is 14.42 $\%, 23.73 \%, 22.70 \%$, and $25.22 \%$, respectively. In general, the Lagrangean based heuristic performs well compared to the simple heuristic. We also find that in less congested network, either with less groups or destinations, the Lagrangean based heuristic outperforms the simple heuristic such as the case A of cellular network and case B of scale-free network.

There are two main reasons of which the Lagrangean based heuristic works better than the simple algorithm. First, the Lagrangean based heuristic makes use of the related Lagrangean multipliers which include the potential cost for routing on each link in the topology. Second, the Lagrangean based heuristic is iteration-based and is guaranteed to improve the solution quality iteration by iteration. Therefore, in a more complicated testing environment, the improvement ratio is higher. To claim optimality, the results show that most of the cases have a gap of less than $40 \%$. We also found that the simple heuristic performs well in many cases, such as the case B of grid network and case D of cellular network.

\section{Conclusions}

In this paper, we attempt to solve the problem of capacitated max-revenue multicast routing and partial 
admission control for multirate multimedia distribution. Our achievement of this paper can be expressed in terms of mathematical formulation and experiment performance. In terms of formulation, we propose a precise mathematical expression to model this problem well. In terms of performance, the proposed Lagrangean based heuristic outperforms the simple heuristics. Our model can be extended to deal with the QoS constrained multicast routing and admission control problem by adding delay constraints. These issues will be addressed in future works.

\section{References}

[1] D. Wu, Y. T. Hou, W. Zhu, T. Q. Zhang, and J. Peha, "Streaming video over the Internet: approaches and directions", IEEE Trans. on Circuits and Systems for Video Technology, vol. 11, no. 3, March 2001, pp. 282300.

[2] G. Karlsson and M. Vetterli, "Packet video and its integration into the network architecture", IEEE JSAC, vol. 7, 1989, pp. 739-751.

[3] E. Amir, S. McCanne, and H. Zhang, "An application level video gateway", Proc. of ACM Multimedia, Nov.
1995, pp. 255-265.

[4] T. Turletti and J. C. Bolot, "Issues with multicast video distribution in heterogeneous packet networks", Proc. of the $6^{\text {th }}$ International Workshop on Packet Video, 1994.

[5] H.C. Cheng and Y. S. Lin, "Minimum-cost multicast routing for multi-layered multimedia distribution", Proc. of IFIP/IEEE MMNS '04, San Diego, CA, USA, Oct. 2004, pp. 102-114.

[6] M. L. Fisher, "The Lagrangian relaxation method for solving integer programming problems", Management Science, vol. 27, 1981, pp. 1-18.

[7] A. M. Geoffrion, "Lagrangean relaxation and its uses in integer programming", Math. Programming Study, vol. 2, 1974, pp. 82-114.

[8] M. Held and R. M. Karp, "The Traveling salesman problem and minimum spanning trees: Part I", Operations Research, vol. 18, 1970, pp. 1138-62.

[9] A. -L. Barabasi and R. Albert, "Emergence of scaling in random networks", Science, vol. 286. Oct. 1999, pp. 509-512.

[10] N. F. Maxemchuk, "Video Distribution on Multicast Networks", IEEE JSAC, vol. 15, No. 3, 1997, pp. 357372.

Table 2. Selected results of computational experiments

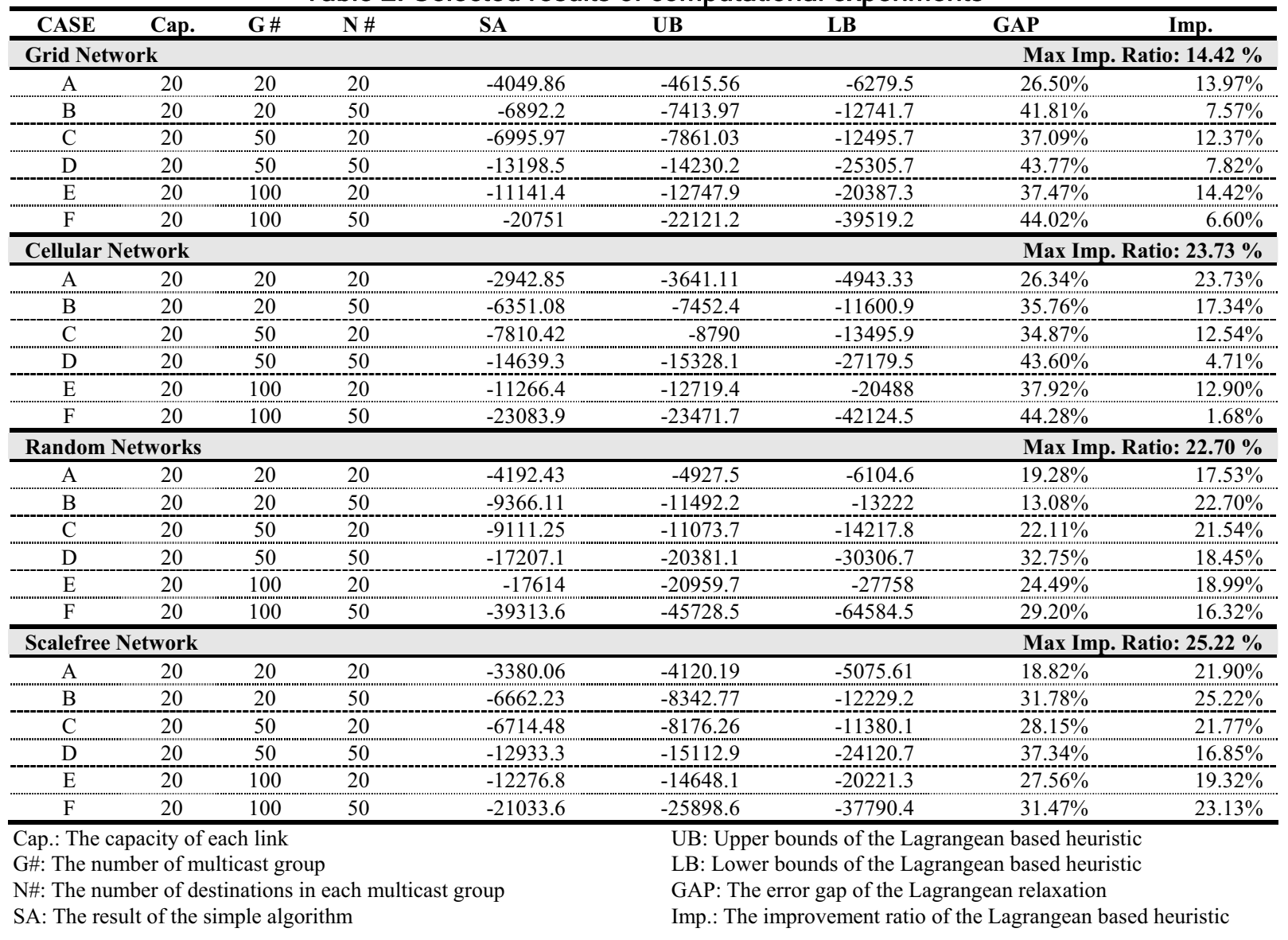

